\title{
Comprehensive methylation analysis in typical and atypical PWS and AS patients with normal biparental chromosomes 15
}

\author{
Maren Runte ${ }^{1}$, Claudia Färber ${ }^{1}$, Christina Lich ${ }^{1}$, Michael Zeschnigk ${ }^{1}$, Tina Buchholz ${ }^{2}$, \\ Arabella Smith $^{2}$, Lionel Van Maldergem ${ }^{3}$, Joachim Bürger ${ }^{4}$, Françoise Muscatelli ${ }^{5}$, \\ Gabriele Gillessen-Kaesbach ${ }^{1}$, Bernhard Horsthemke*,1 and Karin Buiting ${ }^{1}$
}

\begin{abstract}
${ }^{1}$ Institut für Humangenetik, Universitätsklinikum Essen, Essen, Germany; ${ }^{2}$ The New Childreńs Hospital, Sydney, Australia; ${ }^{3}$ Centre de Génétique Humaine, Institute de Pathologie et de Genetique, Loverval, Belgium; ${ }^{4}$ Institut für Humangenetik, Charité, Humboldt-Universität, Berlin, Germany; ${ }^{5}$ Faculté de Médecine, Marseille, France
\end{abstract}

Imprinting defects in 15q11-q13 are a rare but significant cause of Prader-Willi syndrome (PWS) and Angelman syndrome (AS). Patients with an imprinting defect have apparently normal chromosomes 15 of biparental origin, but are recognised by uniparental DNA methylation at D15S63 (PW71) or SNURF-SNRPN exon 1. We have investigated the methylation status of five additional loci in 12 such patients with or without a deletion in the imprinting centre. In each patient, the imprinting defect affected all loci tested. During routine diagnostic testing we identified four patients who had a normal methylation pattern at SNURF-SNRPN exon 1, but an abnormal pattern at D15S63. In two of these patients, who were suspected of having PWS, this change was restricted to D15S63. In two patients suspected of having AS, several but not all loci were affected. Using a newly developed methylation-specific PCR test for D15S63 we found that these methylation changes are rare in patients suspected of having AS. Although we can not prove that the methylation changes in the four patients are causally related to their disease, our findings demonstrate that spatially restricted changes in methylation can occur. In some cases, these changes may reflect incomplete imprint spreading. European Journal of Human Genetics (2001) 9, 519-526.

Keywords: Prader-Willi syndrome; Angelman syndrome; imprinting; DNA-methylation

\section{Introduction}

Prader-Willi syndrome (PWS; MIM 176270) and Angelman syndrome (AS; MIM 105830) are two distinct disorders which are caused by a loss of function of imprinted genes on proximal 15q. At the molecular level, the parental alleles of the PWS/AS region can be distinguished by DNA methylation, and therefore methylation analysis can be used as a diagnostic tool for both syndromes. Although many differentially methylated sites in 15q11-q13 have been identified, typically D15S63 (PW71) and/or SNURF-SNRPN exon 1 are

*Correspondence: Prof Dr Bernhard Horsthemke, Hufelandstraße 55, D-45122 Essen, Germany. Tel: +49 201723 4556; Fax: +49 201723 5900; E-mail: b.horsthemke@uni-essen.de

Received 8 February 2001; revised 30 March 2001; accepted 2 April 2001 investigated. ${ }^{1,2}$ Changes in the parent-specific methylation pattern at these loci are most often due to a large deletion or uniparental disomy (UPD). However, a small group (2-4\%) of patients have apparently normal chromosomes of biparental origin, but uniparental DNA methylation ${ }^{2-8}$ and gene expression. ${ }^{9}$ These patients have an imprinting defect. In some $(10-15 \%)$ of these patients the incorrect epigenotype of one of the chromosomes is caused by a deletion in the imprinting centre (IC). ${ }^{5,9-13}$

Although more extended methylation studies have been performed in special cases, ${ }^{5,10,12}$ no systematic investigation of the methylation pattern at different loci in patients with an imprinting defect has been reported so far. Therefore, it was not clear (i) whether the imprinting defect affected only some loci or the whole imprinted domain, and (ii) whether the affected chromosomes were imprinted in the opposite 
direction or uniformly methylated and unmethylated, respectively. To address these questions, we performed a comprehensive methylation analysis in 12 AS and PWS patients with an imprinting defect by investigating loci which are normally methylated on the maternal allele and loci which are normally methylated on the paternal allele.

Furthermore, we studied four patients suspected of having PWS or AS who by routine diagnostic testing had been found to have normal SNURF-SNRPN, but abnormal D15S63 methylation. In the course of this work we have developed a methylation-specific PCR (MSP) test for D15S63. This test may be useful to confirm results obtained by methylation analysis of the SNURF-SNRPN gene, which is the most suitable locus for testing patients.

\section{Patients}

\section{Patients with an imprinting defect}

Twelve patients with an imprinting defect were seen by experienced clinicians and diagnosed as having typical AS or PWS. Patients AS1 and AS6 were described by Bürger et al. ${ }^{6}$ (AS-La and AS-D). Patients AS2, 3 and 4 were described by Buiting et al. ${ }^{7}$ where they are referred to as ASID-04, ASID-06 and ASID-10. One of the AS patients with an imprinting defect (AS5) was described in Ohta et al. ${ }^{11}$ (case ASF).

The first five patients with PWS and a sporadic imprinting defect (PWS1-5) were reported in Buiting et al, ${ }^{7}$ named PWSID-02, PWSID-05, PWSID-06, PWSID-07 and PWSID-12. The PWS patient with an IC-deletion (PWS6) was described in Ohta et al. ${ }^{12}$ (PWS-P).

\section{Patients with an abnormal methylation pattern}

By routine diagnostic testing for AS and PWS we identified four patients who had a normal SNURF-SNRPN, but abnormal D15S63 methylation.

Patient LE The proposita was born at term as the first child of a non-consanguineous German couple. A younger brother is developing normally. Birth weight of the patient was $2510 \mathrm{~g}$ ( $-2.8 \mathrm{SD})$, length $47 \mathrm{~cm}(-3.2 \mathrm{SD})$ and occipitofrontal circumference (OFC) was $31.5 \mathrm{~cm}$ (-2.6 SD). During the neonatal period she had feeding problems but no severe muscular hypotonia. The developmental milestones were delayed. She began to walk at the age of 2 years and to speak at the age of 4 years. Since the age of 2 years, she is obese and does not feel satiety. She was referred to a clinical genetics department (GG-K) at the age of 5 years and 10 months with the tentative diagnosis of Prader - Willi syndrome. Her height was $108.4 \mathrm{~cm}$ (3-10th percentile). She was severely obese with a weight of $24.5 \mathrm{~kg}$ ( $>97 \mathrm{th}$ percentile). OFC of $50.5 \mathrm{~cm}$ corresponded to the 25 th percentile. She was mentally retarded and showed general obesity but no muscular hypotonia. Facial features were normal. Apart from clinodactyly of digitus $\mathrm{V}$ she had no characteristic dysmorphic signs. By microsatellite analyses, a common large deletion and a UPD could be excluded. Cytogenetic analysis showed a normal karyotype $(46, \mathrm{XX})$.

Patient FB The patient was born as the 4th child of a healthy nonconsanguineous couple. The three sibs are normal and healthy. The propositus was born at term with a birth weight of $4300 \mathrm{~g}(+1.7 \mathrm{SD})$, and a length of $55 \mathrm{~cm}$ (+0.6 SD). During pregnancy the mother noticed reduced foetal movements. The boy had neither neonatal feeding problems nor muscular hypotonia. Sleep problems were reported during the first 2 years of life. He began to walk at 19 months and to speak at about 3 years and 6 months. The mentally retarded boy was examined (GG-K) at the age of 8 years and 9 months. His height was $149 \mathrm{~cm}$ ( $>97$ th percentile) and he was markedly obese with a weight of $56 \mathrm{~kg}$ ( $>97 \mathrm{th}$ percentile). His bone age was accelerated (12 years) and he had developed signs of a precocious puberty. No dysmorphic signs were present. He tended to be aggressive. Microsatellite analysis showed a normal biparental inheritance.

Patient MK The patient was born in August 1984 after a normal pregnancy as the first child to healthy unrelated parents with an unremarkable family history. He was delivered by caesarean section as a floppy baby. Breast feeding was impossible, and he regurgitated for the first 6 months. He was first seen by a paediatrician after 8 days. He had delayed milestones and began to walk just 3 weeks before his second birthday. Furthermore speech was missing and he had an L convergent squint. One of his testes was undescended. He has a high palate as well as blue eyes and brown hair and is not hypopigmented. His 5th fingers show bilateral clinodactyly. When seen in 1993 he had sleep problems, but had no signs of epilepsy. He had an ataxic gait. Furthermore, he had a large mouth and a big chin. He was a happy child that laughed a lot and inappropriately. He was able to understand and used a basic sign language with about 15 words. He was toilet trained. His weight was $35 \mathrm{~kg}$ (90th percentile), his height $130.4 \mathrm{~cm}$ (25-50th percentile), and his head circumference was $55.5 \mathrm{~cm}$ (nearly 98th percentile). When he was last seen in 1996 his puberty developed normally and he still did not present any sign of epilepsy. His head circumference and height were still above the 90th percentile. Exept for the large head circumference the patient showed typical features for AS. Microsatellite analysis showed normal biparental inheritance and also excluded a typical deletion. This was confirmed by fluorescence-in situ-hybridisation.

\section{Patient MC}

The proposita was born in 1950 to unrelated Caucasian parents. Her mother was 28 years old at the time of delivery, which was unremarkable. She had had two miscarriages before. From the age of 6 months, the patient had episodes of generalised seizures. Developmental milestones and speech 
were delayed. She began to walk at 24 months and was severely mentally retarded. She showed signs of primary thrombocythemia (platelet count between 700 and 800 000/ $\mathrm{mm}^{3}$ ) and polyglobulism with thromboembolic events (lower limbs thrombosis, pulmonary embolism, myocardial infarction). She still has recurrent seizures, which are very difficult to control with antiepileptic drugs. On clinical examination at age 41, her height was $153 \mathrm{~cm}(-2 \mathrm{SD})$ and OFC was $54 \mathrm{~cm}$. There is midface hypoplasia, downslanting palpebral fissures and telecanthus, low set ears, flat occiput and bilateral epicanthus. On slit lamp examination of the eye, she showed a bilateral cataract and a normal fundus. Cytogenetic analysis of peripheral lymphocytes presented a normal 46,XX karyotype.

\section{Methods}

\section{DNA methylation analysis by Southern blotting}

DNA from peripheral blood ( $2 \mu \mathrm{g}$ each) was digested with the appropriate restriction enzymes. The fragments were resolved on 0.7 to $1.0 \%$ agarose gels and transferred to Biodyne A nylon membranes (PALL, Portsmouth, UK). All Southern blots were performed in duplicates. Probes were labelled by random oligonucleotide priming and $\left[\alpha_{-}{ }^{32} \mathrm{P}\right] \mathrm{CTP}$ (NEN, Boston, MA, USA). Autoradiography was performed at $-80^{\circ} \mathrm{C}$ with intensifying screens and Fuji films. For patients $\mathrm{LE}, \mathrm{FB}, \mathrm{MK}$ and MC, relative signal intensities (Figure 3) were determined by scanning densitometry with a Shimadzu C9000 densitometer.

For methylation analysis in 15q11-q13 the following probes were used: a cDNA clone ${ }^{14}$ or a PCR derived probe generated with primers RN700 and RN709 (kindly provided by RD Nicholls) for the NDN locus, probe B800 and D800 for $\mathrm{u} 1 \mathrm{D}$ and $\mathrm{u} 1 \mathrm{~B}$, respectively, ${ }^{15}$ probe $71.19 .12 \mathrm{HR},{ }^{9} \mathrm{PW} 71 \mathrm{C}$ for the CfoI site at locus PW71/u1A as well as for the HpaII site in the LTR at this locus, YR9AB (also termed RN4RR, ${ }^{16-18}$ Y48.5, ${ }^{5} \mathrm{~KB} 17$ for the SNURF-SNRPN CpG-island, ${ }^{9}$ Smn1 for locus SNURF-SNRPN intron $7^{19,20}$ and YL48E (K Buiting, unpublished). For the $K v D M R 1$-locus on chromosome 11p15 a PCR probe was used amplified with primers DMRP2F and DMRP2R. ${ }^{21}$

Exclusion of structural rearrangements or deletions of the IC region was performed using PCR generated probes Snpwsct2a (309 bp), Snpwsct5a (131 bp), Snpwsct9a (531 bp), and Snpwsct12a (1.2 kb). Each probe was hybridised onto EcoRI, BamHI, HindIII, XbaI, and ScaI digested DNA of the patients. These probes were generated using following primers: Snpwsct2aF 5'-TTTGCACCTAATCTCCCACTG-3', Snpwsct2aR 5'-CTAGGCCCTTGGTCCAAAT-3', Snpwsct5aF 5'-CAGTATT TCAAATGTGACTTGTAGGA-3', Snpwsct5aR 5'-AAATCTCACCTCTTTTTCACTCA-3', Snpwsct9aF 5'-AGTCCTGAATCCAGAACATGA-3', Snpwsct9aR 5'-CAAAATAATGTGAAGTTTTCCCTGTA-3', Snpwsct12aF 5'-TGAATAGCCAGTGTTTTTGAGTT-3', Snpwsct12aR 5'-TACACCTACCTGCGCTCAAC-3'.
Methylation-specific PCR (MSP) for the D15S63 locus Bisulfite treatment of genomic DNA and methylationspecific PCR at the SNURF-SNRPN locus was performed as described by Zeschnigk et al. ${ }^{22}$ D15S63 MSP was performed under the same conditions as described for the SNURF-SNRPN MSP, except that the concentration of each primer was adjusted to $0.4 \mu \mathrm{M}$ and an annealing temperature of $55^{\circ} \mathrm{C}$ was used. At this temperature a rare single nucleotide variant in the binding site of the mat and pat primers (estimated frequency $<0.01$; Buiting et al, unpublished) does not confound the test. PCR products were analysed on 3\% agarose gels. Primer sequences were as follows: PW 71 mat $23 \mathrm{C}:\left(5^{\prime}-[\mathrm{C}]_{23}\right.$ GATCGTATT TAT G GTA TAATTGCGTTTGC-3'), PW71com: (5'-AATATCAACACTCCTCATTAAAATTCCCTCC-3') and PW71pat: (5'GAGGATGATTGTATTTATGGTATAATTGTGTTTGT-3').

\section{Results and discussion \\ Methylation analysis in patients with an imprinting defect}

We studied six PWS and six AS patients. One PWS and two AS patients had an inherited deletion in the imprinting centre. The other nine patients had a sporadic imprinting defect. The clinical diagnoses had been confirmed by methylation analysis of SNURF-SNRPN exon 1 and/or PW71/u1A (D15S63). We examined five additional loci (from cen to tel): $N D N,{ }^{14,23} \mathrm{u} 1 \mathrm{D},{ }^{24} \mathrm{u} 1 \mathrm{~B},{ }^{24,25}$ SNURF-SNRPN intron $7^{20,26}$ and YL48E (K Buiting, unpublished). These loci include two sites which are normally methylated on the maternal chromosome $(N D N, \mathrm{u} 1 \mathrm{~B})$ and three sites which are preferentially methylated on the paternal chromosome (u1D, SNURFSNRPN intron 7, YL48E). The results are shown in Figure 1 and summarised in Figure 2. We found that in most cells all loci were incorrectly methylated: In the AS patients, the maternal chromosome was unmethylated at sites that should be methylated, and methylated at sites that should be unmethylated. The reciprocal findings were made on the paternal chromosome of PWS patients. These results indicate that the defect affects the whole imprinted domain and that the affected chromosomes are imprinted in the opposite direction rather than methylated or unmethylated. There was no difference between patients with an IC deletion or a sporadic imprinting defect.

\section{Detection of unusual methylation patterns}

In the course of routine diagnostic testing we identified four patients who had a normal methylation pattern at SNURFSNRPN exon 1, but an abnormal pattern at D15S63. Two of the patients (LE und FB) had a weak maternal band and a strong paternal band at the $C f o$ I site within exon $\mathrm{u} 1 \mathrm{~A}$, ie an AS-like pattern, although they were suspected of having PWS (Figure 3). The other two patients (MK and MC) had a strong maternal band and a faint paternal band at the CfoI-site, ie a PWS-like pattern, although they were suspected of having AS 

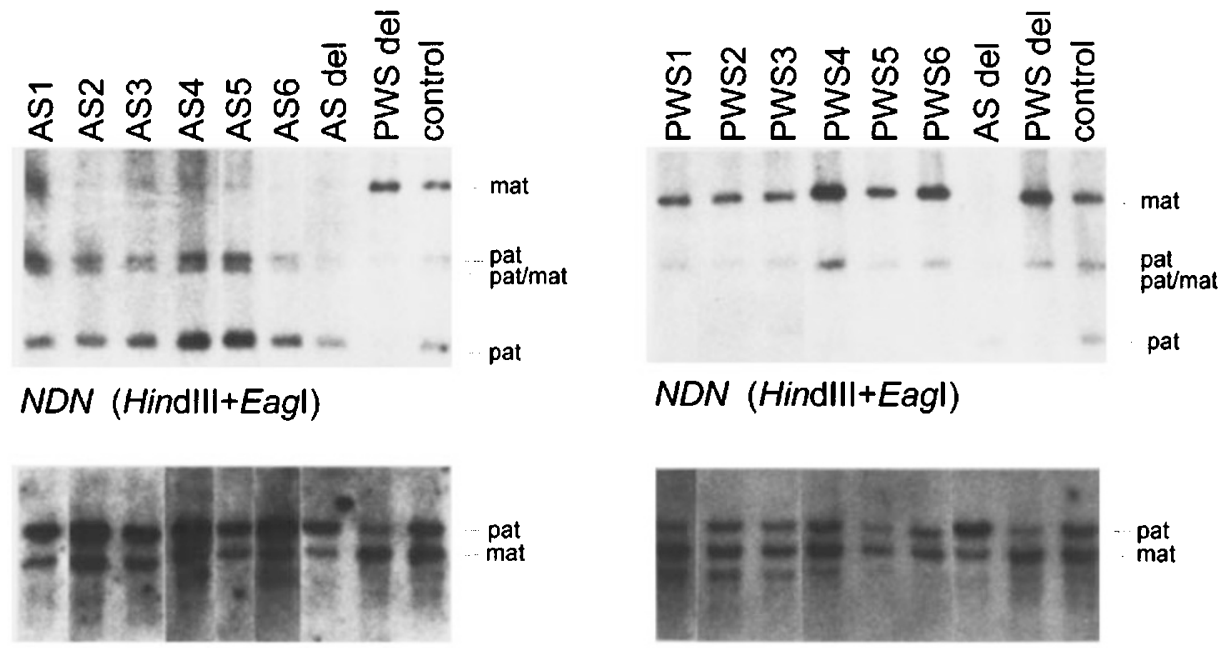

u1D (HindllI+Cfol)

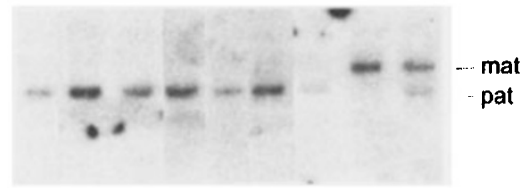

u1B (HindIII+Cfol)

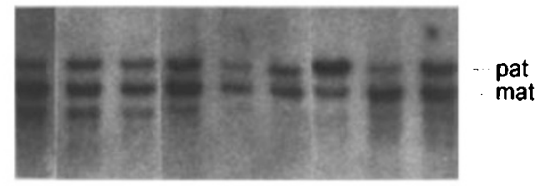

u1D (HindIII+Cfol)

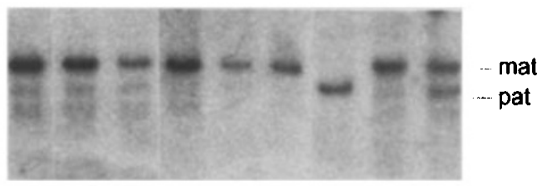

u1B (HindIII+Cfol)

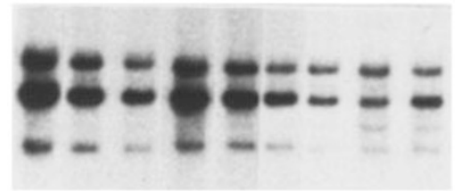

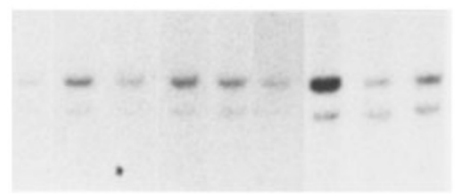

YL48E (HindIII+Hpall)

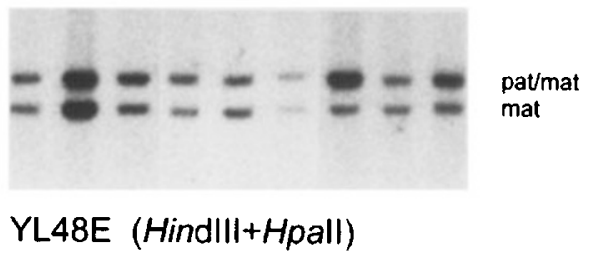

Figure 1 Methylation analysis of patients with an imprinting defect. Patients AS4, AS6 and PWS 6 have an IC deletion, whereas the other patients have a sporadic imprinting defect. PWS del and AS del represent patients with a de novo common large deletion of $15 \mathrm{q} 11$-q13. The fragment sizes of the paternal (pat) and maternal (mat) alleles are as follows: NDN $2.7 \mathrm{~kb}$ (mat), $1.74 \mathrm{~kb}$ (pat), $1.7 \mathrm{~kb}$ (mat/pat), $0.9 \mathrm{~kb}$ (pat); u1D: $13.0 \mathrm{~kb}$ (pat), $9.0 \mathrm{~kb}$ (mat); u1B: $9.2 \mathrm{~kb}$ (mat), $7.4 \mathrm{~kb}$ (pat), $1.6 \mathrm{~kb}$ (pat; not shown); for SNRPN intron 7: 7.5 kb (SNRPNP1; SNURF-SNRPN pseudogene on chromosome 6), $5.8 \mathrm{~kb}$ (mat/pat), $5.0 \mathrm{~kb}$ (mat), $4.5 \mathrm{~kb}$ (exon 3); YL48E: $3.0 \mathrm{~kb}$ (mat/pat), $2.6 \mathrm{~kb}$ (mat). All the AS patients have a faint or absent maternal band whereas all the PWS patients have a faint or absent paternal band.

(Figure 3). None of the four patients had a deletion of $D 15 S 63$ (data not shown), which occurs as a rare neutral variant and may have explained these findings. ${ }^{27,28}$ Likewise, we excluded other structural rearrangements in this region by
Southern blot analysis with a battery of probes covering about $35 \mathrm{~kb}$ of genomic DNA of the IC (data not shown).

To find out whether the aberrant methylation pattern in the four patients was restricted to D15S63, we investigated 
methylated

unmethylated

normal

control

ID/PWS

ID/AS

LE

FB
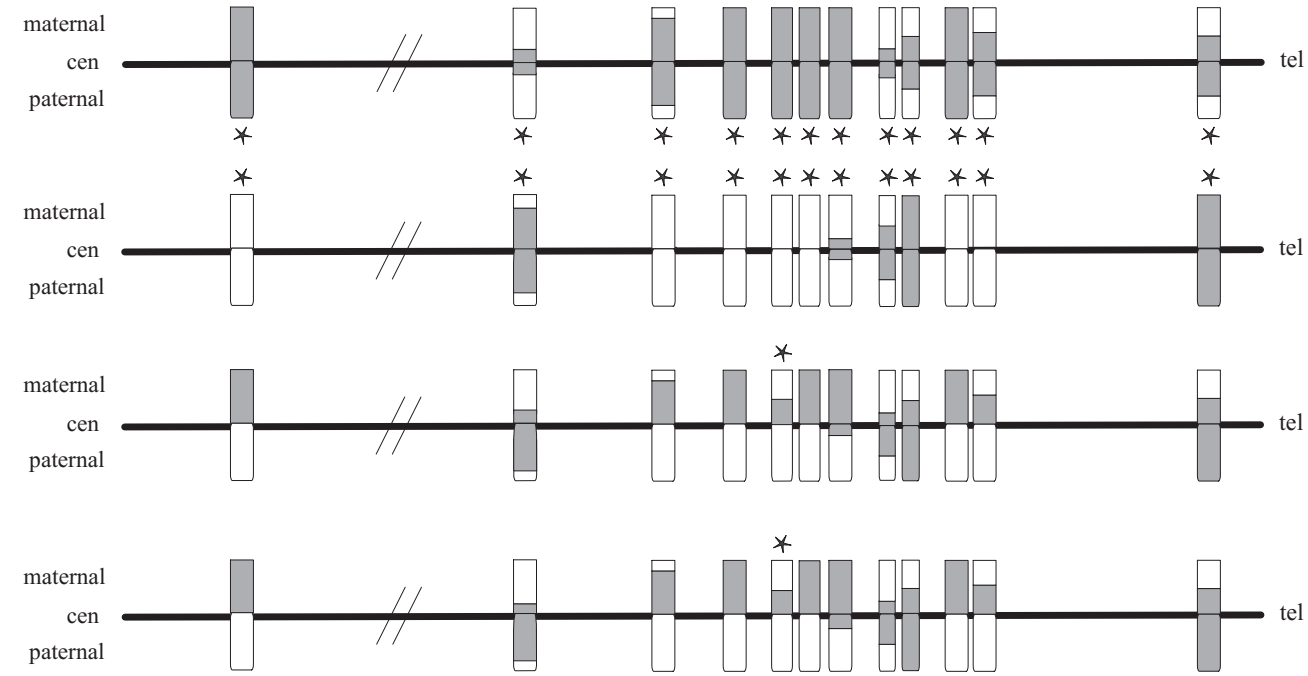

MK

maternal

maternal
cen
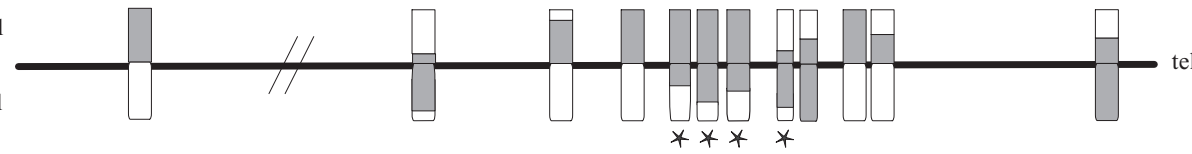

MC

not drawn to scale

Figure 2 Schematic representation of the methylation status in the PWS and AS patients with an imprinting defect (ID) and the patients $\mathrm{LE}, \mathrm{FB}, \mathrm{MK}$ and MC. U1D, u1B and U1A are alternative $5^{\prime}$ exons of the IC/SNRPN-transcripts. ${ }^{24,25}$ The boxes show the degree of methylation in grey. Stars indicate an aberrant pattern. In patient MC, the parental identity of the aberrant pattern at loci Y48.5 and YL48E is unclear (indicated by question marks).

the loci mentioned above plus 71.19 .12 (B Dittrich, unpublished), the long terminal repeat (LTR) at locus PW71 ${ }^{1}$ and YR9AB $^{18}$ (Figure 3), which are methylated on the maternal chromosome. Patients LE and FB had a normal biparental methylation pattern at each locus except for the CfoI site mentioned above. Interestingly, the HpaII site within the D15S63 locus, which was mapped $1.3 \mathrm{~kb}$ distal to the $C f o$ I site, was normal. In the parents of LE and FB, the $C f o I$ site was normally methylated (data not shown).
In patient MK we found that four sites were affected: one HpaII site detected by probe Y48.5, one HpaII site detected by probe YR9AB, and the HpaII and CfoI site detected by probe PW71 (D15S63). All four sites were hypermethylated on the paternal chromosome. The aberrantly methylated region spans approximately $50 \mathrm{~kb}$. In the parents, these sites were normally methylated (data not shown).

In patient MC we found that eight sites were affected. While YR9AB was hypomethylated on the maternal allele, 


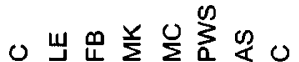

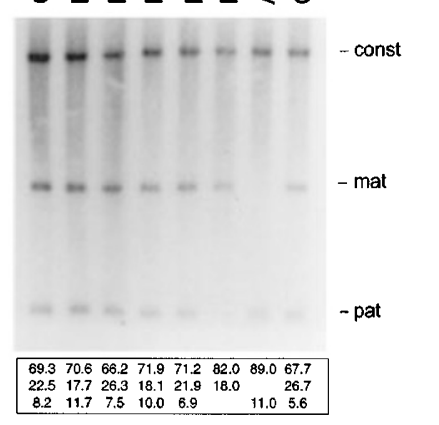

NDN (HindIII + Eagl)

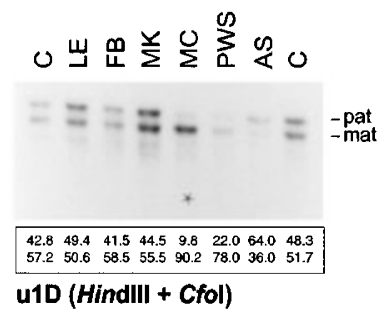

11D (HindIII + Cfol)
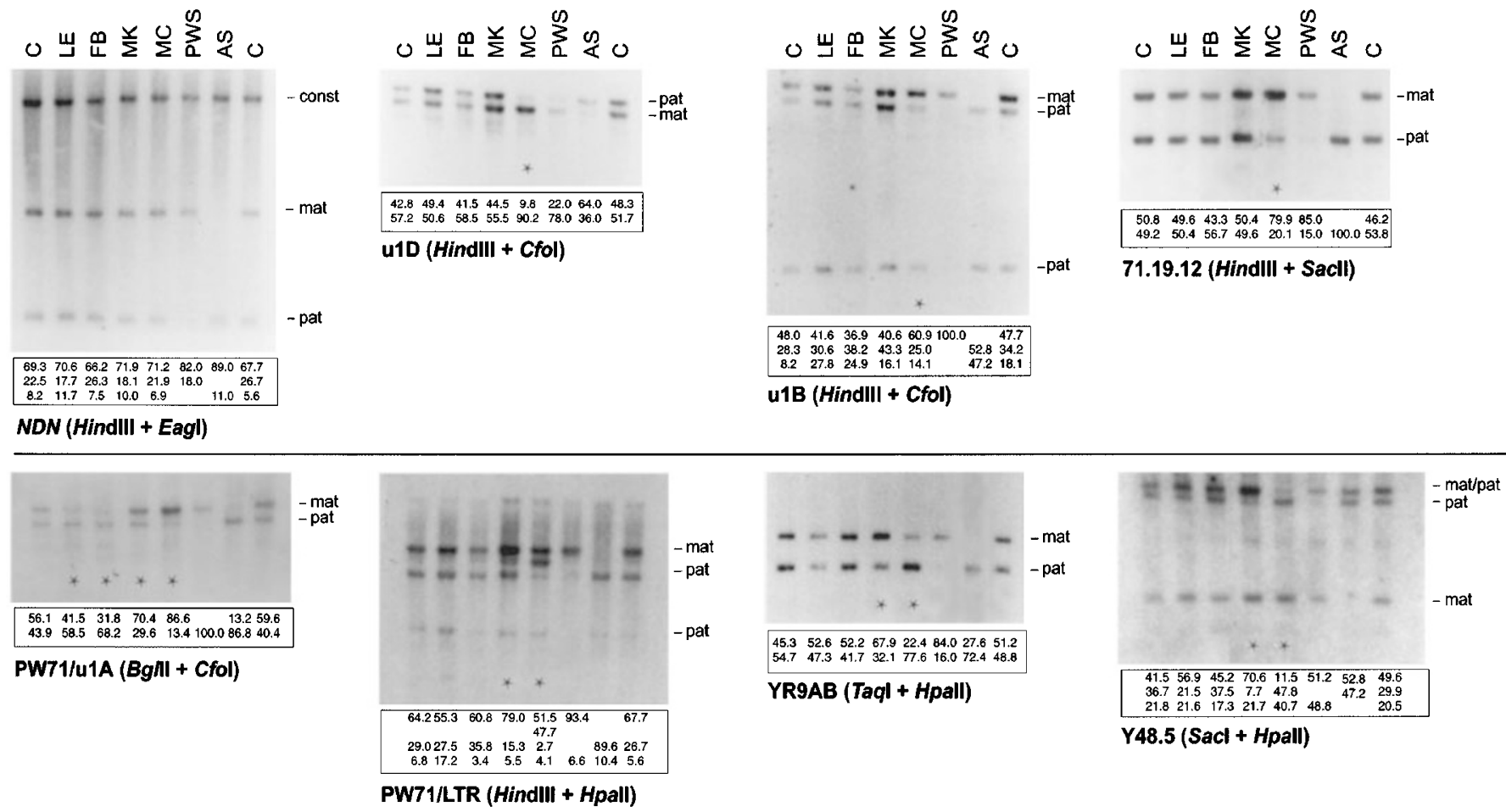

PW71/LTR (HindIII + Hpall)
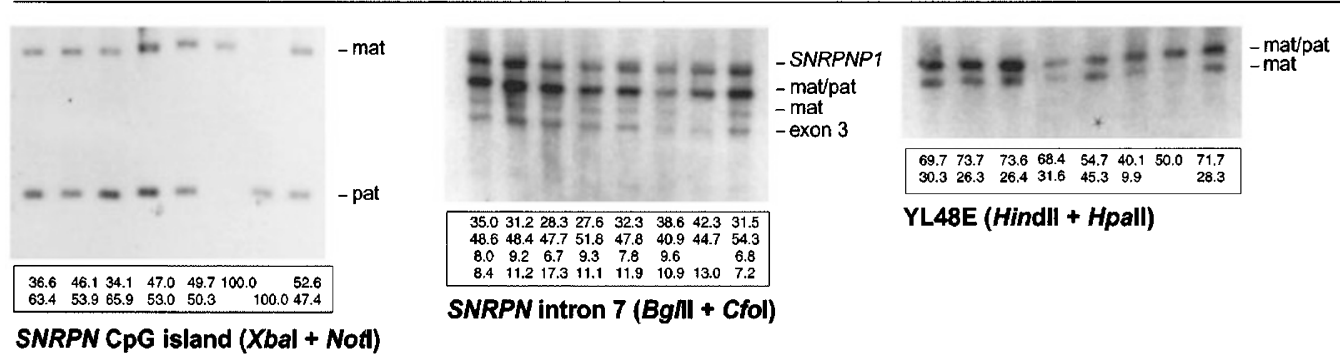

Figure 3 Methylation analysis of patients suspected of having PWS and AS. Each experiment was done in duplicate. One set of Southern blots is shown in this figure. C, normal control; PWS, patient with a paternal deletion of 15q11-q13; AS, patient with a maternal deletion of 15q11-q13. The sizes of the maternal (mat) and paternal (pat) bands for NDN, u1D, u1B, SNRPN intron7 and YL48E are given in Figure 1. 71.19.12: $12.0 \mathrm{~kb}$ (mat), $7.0 \mathrm{~kb}$ (pat); PW71/u1A: $8.0 \mathrm{~kb}$ (mat), $6.4 \mathrm{~kb}$ (pat); PW71/LTR: 6.6 kb (mat), 5.8 kb (additional band in the pattern of MC), $4.4 \mathrm{~kb}$ (pat), $2.5 \mathrm{~kb}$ (pat); YR9AB: $2.2 \mathrm{~kb}$ (mat), $1.8 \mathrm{~kb}$ (pat); Y48.5: $2.8 \mathrm{~kb}$ (mat/pat), $2.5 \mathrm{~kb}$ (mat), $1.0 \mathrm{~kb}$ (pat), SNRPN CpG island: $4.2 \mathrm{~kb}$ (mat), $0.9 \mathrm{~kb}$ (pat). The additional $5.8 \mathrm{~kb}$ Hindlll+Hpall band detected by probe PW71C in the DNA from patient MC is probably due to a DNA polymorphism. The relative intensities of the bands are given below each autoradiogram. Asterisks indicate an aberrant pattern of the patients LE, FB, MK and MC. const, constant band. SNRPNP1, pseudogene of SNURF-SNRPN on chromosome 6.

the two D15S63 restriction sites, 71.19 .12 and $\mathrm{u} 1 \mathrm{~B}$ were hypermethylated on the paternal allele. u1D was hypomethylated on the paternal allele. We also observed methylation changes at Y48.5 and YL48E, but were unable to resolve which chromosome was affected. This is due to the fact that in normal controls differential methylation at these two loci is not as complete as at other loci. Apart from SNURFSNRPN, only NDN was normal.

The aberrant methylation affects seven loci centromeric to SNURF-SNRPN spanning $140 \mathrm{~kb}$, and one locus which maps $200 \mathrm{~kb}$ telomeric to SNURF-SNRPN (YL48E). Un- fortunately, no parental DNA samples were available for testing.

To find out whether aberrant methylation in the four patients might be due to a trans-acting defect affecting other imprinted regions as well, we studied a NotI site at the maternally methylated CpG island (KvDMR1) within intron 10 of the KvLQT1 locus on 11p15, which is often affected in patients with Beckwith-Wiedemann syndrome. ${ }^{21,29}$ We observed a normal pattern in each patient (data not shown).

Next we asked how frequent methylation changes outside SNURF-SNRPN might be. For this, we investigated 48 patients 
A

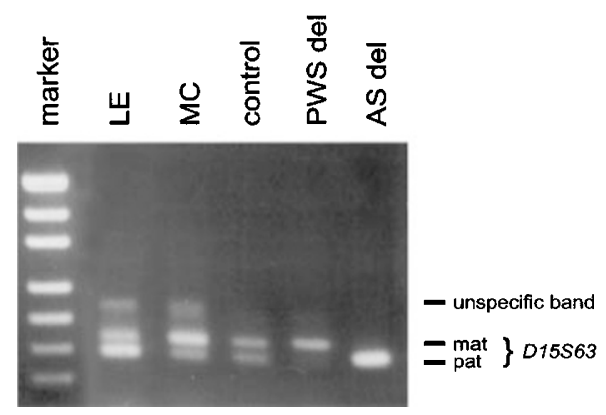

B

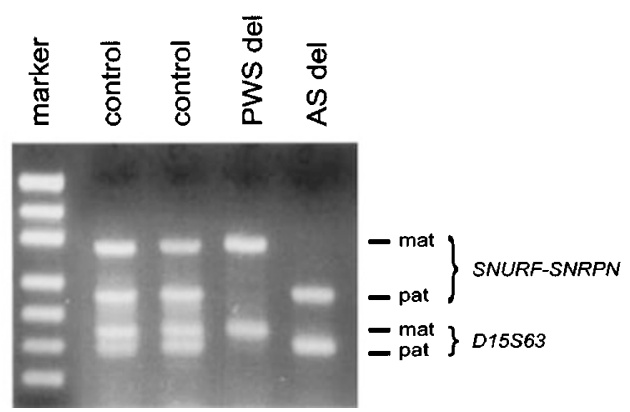

Figure 4 Methylation-specific PCR. (A) Methylation-specific PCR analysis of the D15S63 locus in the patients LE and MC. The other lanes contain DNA from a normal control and from patients with a paternal (PWS del) or maternal (AS del) deletion of 15q11-q13, respectively. The maternal band (mat) is a $161 \mathrm{bp}$ product and the paternal band (pat) is a $143 \mathrm{bp}$ product. In accordance to the Southern blot data (Figure 3) the locus is hypomethylated in patient LE and hypermethylated in patient MC. (B) Multiplex analysis of SNURF-SNRPN and D15S63. The sizes of the SNURF-SNRPN products are $313 \mathrm{bp}$ and $221 \mathrm{bp}$. The sizes of the D15S63 products are as given above.

with normal SNURF-SNRPN methylation who had been suspected of having AS, but who did not have a UBE3A mutation. We developed a methylation-specific PCR (MSP) test for the CfoI site at D15S63. The density of CpG dinucleotides in this region is much lower than in the SNURF-SNRPN exon 1 region. After bisulfite modification there are only two nucleotides, which can be used to distinguish the parental alleles. ${ }^{30}$ PCR is initiated with a primer (PW71com) annealing to a site which is identical in both alleles. Methylation specific primers were designed to specifically bind to the paternal strand (PW71pat) and the maternal strand (PW71mat23C), respectively, both with their 3 ' end annealing to the differentially methylated $C f o$ I site. To distinguish between the parental alleles in a single tube MSP assay, primer PW71mat23C was designed to have a $5^{\prime}$ tail of 23 cytosines, resulting in a maternal MSP product of $161 \mathrm{bp}$ and a paternal product of $143 \mathrm{bp}$ in length. The test was validated by investigating normal controls and patients who had been typed by Southern blot analysis (Figure $4 \mathrm{a}$ ). The primers can be combined with the previously published SNURF-SNRPN MSP primers ${ }^{22}$ in a multiplex assay (Figure $4 \mathrm{~b}$ ). All of the 48 patients had a normal pattern (data not shown), suggesting that methylation changes outside of SNURFSNRPN in this class of patients are rare.

What is the significance of our findings? We cannot prove that the unusual methylation changes in the four patients are related to their disease, especially since we found an 'AS-like' methylation pattern in two patients suspected of having PWS and a 'PWS-like' methylation pattern in two patients suspected of having AS. The first two patients (LE and FB) have only few features of PWS. In these patients, the methylation change is restricted to one site at the D15S63 locus and may reflect a change in the clonal complexity of peripheral blood. LaSalle et al $^{31}$ have recently shown that the methylation pattern at this locus is not strictly maintained within individual cell clones and displays clonal hetero- geneity. Despite clonal variation in D15S63 methylation, peripheral blood is usually very complex due to the generation of diversity for its immune function. It is possible that this complexity and not the imprint itself is disturbed in the two patients. The same argument may apply to the other two patients, but in these patients other loci in addition to D15S63 are affected. Whereas patient MC has only few features of AS, patient $\mathrm{MK}$ is rather typical. It is possible that in these cases imprinting was indeed affected. One may speculate that the imprint was correctly switched at the imprinting centre, but that the spreading was incomplete.

\section{Acknowledgments}

We thank Dr Eamonn Maher for providing us a DNA sample of a Beckwith-Wiedemann patient with an aberrant methylation pattern at KVDMR1 (11p15), Dr Dirk Prawitt for oligonucleotides for amplifying the probe DMRP, Dr Robert D Nicholls for NDN-primer sequences, and Dr Amy Lossie for hybridisation conditions for the NDN-locus. We also thank Dr Paul Jenkins, Paediatrician, Canberra, Australia and Dr Penny Johnson, Developmental Paediatrician, Canberra, Australia. They have both been very helpful with arranging appointments and follow up with the family $M K$, and providing a lot of background information. We also thank Dr Andreas Weinhäusel for sharing with us his experience with the D15S63 MSP test. Part of this work was supported by the Deutsche Forschungsgemeinschaft.

\section{References}

1 Dittrich B, Buiting K, Groß S, Horsthemke B: Characterization of a methylation imprint in the Prader-Willi syndrome chromosome region. Hum Mol Genet 1993; 2: 1995 - 1999.

2 Sutcliffe JS, Nakao M, Christian S et al: Deletions of a differentially CpG island at the SNRPN gene define a putative imprinting control region. Nat Genet 1994; 8: 52-58.

3 Glenn CC, Nicholls RD, Saitoh S et al: Modification of 15q11q13 DNA methylation imprints in unique Angelman and Prader-Willi patients. Hum Mol Genet 1993; 2: 1377-1382. 
4 Reis A, Dittrich B, Greger V et al: Imprinting mutations suggested by abnormal DNA methylation patterns in familial Angelman and Prader-Willi syndromes. Am J Hum Genet 1994; 54: $741-747$.

5 Buiting K, Saitoh S, Groß S et al: Inherited microdeletions in the Angelman and Prader-Willi syndromes define an imprinting center on human chromosome 15. Nat Genet 1995; 9: 395-400.

6 Bürger J, Buiting K, Dittrich B et al: Different mechanisms and recurrence risks of imprinting defects in Angelman syndrome. Am J Hum Genet 1997; 61: 88-93.

7 Buiting K, Dittrich B, Groß S et al: Sporadic imprinting defects in Prader-Willi syndrome and Angelman syndrome: implications for imprint-switch models, genetic counseling, and prenatal diagnosis. Am J Hum Genet 1998; 63: 170-180.

8 Gillessen-Kaesbach G, Demuth S, Thiele H, Theile U, Lich C, Horsthemke B: A previously unrecognised phenotype characterised by obesity, muscular hypotonia, and ability to speak in patients with Angelman syndrome caused by an imprinting defect. Eur J Hum Genet 1999; 7: 638-644.

9 Saitoh S, Buiting K, Rogan PK et al: Minimal definition of the imprinting center and fixation of a chromosome 15q11-q13 epigenotype by imprinting mutations. Proc Natl Acad Sci USA 1996; 93: 7811-7815.

10 Schuffenhauer S, Buchholz T, Stengel-Rutkowski S, Buiting K, Schmidt H, Meitinger T: A familial deletion in the Prader-Willi syndrome region including the imprinting control region. Hum Mut 1996; 8: 288-292.

11 Ohta T, Buiting K, Kokkonen H et al: Molecular mechanisms of Angelman syndrome in two large families involves an imprinting mutation. Am J Hum Genet 1999; 64: 385 - 396.

12 Ohta T, Gray TA, Rogan PK et al: Imprinting-mutation mechanisms in Prader-Willi syndrome. Am J Hum Genet 1999; 64: $397-413$.

13 Buiting K, Färber C, Kroisel P et al: Imprinting centre deletions in two PWS families: implications for diagnostic testing and genetic counselling. Clin Genet 2000; 58: 284-290.

14 Jay P, Rougeulle C, Massacrier A et al: The human necdin gene, NDN, is maternally imprinted and located in the Prader-Willi syndrome chromosomal region. Nat Genet 1997; 17: 357-361.

15 Bielinska B, Blaydes SM, Buiting K et al: De novo deletions of SNRPN exon 1 in early human and mouse embryos result in a paternal to maternal imprint. Nat Genet 2000; 25: 74-78.

16 Dittrich B, Buiting K, Groß S, Horsthemke B: An insertion/ deletion polymorphism at the D15S63 locus in the critical Prader-Willi syndrome region in 15q11-13. Hum Genet 1994; 94: 583-584.

17 Gabriel J, Gottlieb W, Garcia A, Rogan PK, Saitoh S, Nicholls RD: A common insertion/deletion polymorphism in the PraderWilli syndrome minimal critical region. Hum Mol Genet 1994; 3: 1912.
18 Nicholls RD, Saitoh S, Horsthemke B: Imprinting in Prader-Willi and Angelman syndromes. TIG 1998; 14: 194-200.

19 Buiting K, Dittrich B, Groß S et al: Molecular definition of the Prader-Willi syndrome chromosome region and orientation of the SNRPN gene. Hum Mol Genet 1993; 2: 1991-1994.

20 Glenn CC, Porter KA, Jong MT, Nicholls RD, Driscoll DJ: Functional imprinting and epigenetic modification of the human SNRPN gene. Hum Mol Genet 1993; 2: 2001 - 2005.

21 Smilinich NJ, Day CD, Fitzpatrick GV et al: A maternally methylated CpG island in KvLQT1 is associated with an antisense paternal transcript and loss of imprinting in Beckwith-Wiedemann syndrome. Proc Natl Acad Sci USA 1999; 96: 8064-8069.

22 Zeschnigk M, Lich C, Buiting K, Doerfler W, Horsthemke B: A single-tube PCR test for the diagnosis of Angelman and PraderWilli syndrome based on allelic methylation differences at the SNRPN locus. Eur J Hum Genet 1997; 5: 94-98.

23 MacDonald HR, Wevrick R: The necdin gene is deleted in PraderWilli syndrome and is imprinted in human and mouse. Hum Mol Genet 1997; 6: 1873-1878.

24 Färber C, Dittrich B, Buiting K, Horsthemke B: The chromosome 15 imprinting centre (IC) region has undergone multiple duplication events and contains an upstream exon SNRPN that is deleted in all Angelman syndrome patients with an IC. Hum Mol Genet 1999; 8: 337-343.

25 Dittrich B, Buiting K, Korn B et al: Imprint switching on human chromosome 15 may involve alternative transcripts of the SNRPN gene. Nat Genet 1996; 14: 163-170.

26 Özcelik T, Leff S, Robinson W, Donlon T et al: Small nuclear ribonucleoprotein polypeptide $\mathrm{N}$ (SNRPN), an expressed gene in the Prader-Willi syndrome critical region. Nat Genet 1992; 2 : $265-269$.

27 Buiting K, Dittrich B, Dworniczak B et al: A 28-kb deletion spanning D15S63 (PW71) in five families: a rare neutral variant? Am J Hum Genet 1999; 65: 1588 - 1594.

28 Silverstein S, Lerer I, Buiting K, Abeliovich D: The 28-kb deletion spanning D15S63 is a polymorphic variant in the Ashkenazi Jewish Population. Am J Hum Genet 2001; 68: 261-263.

29 Engel JR, Smallwood A, Harper A et al: Epigenotype-phenotype correlations in Beckwith-Wiedemann syndrome. J Med Genet 2000; 37: $921-926$.

30 Zeschnigk M, Schmitz B, Dittrich B, Buiting K, Horsthemke B, Doerfler W: Imprinted segments in the human genome: Different DNA methylation patterns in the Prader-Willi/Angelman Syndrome region as determined by the genomic sequencing method. Hum Mol Genet 1997; 6: 387 - 395.

31 LaSalle JM, Ritchie RJ, Glatt H, Lalande M: Clonal heterogeneity at allelic methylation sites diagnostic for Prader-Willi and Angelman. Proc Natl Acad Sci USA 1998; 95: 1675 - 1680. 